\title{
Evaluación de impacto ambiental en la mina artesanal de arcilla, santa cruz en el municipio de manaure, la guajira.
}

\author{
Evaluation of the Environmental Impact in the handcraft \\ Clay mine,Santa Cruz,Manaure, La Guajira
}

\author{
Danny Daniel López Juvinao \\ Ingeniero en Minas, Magister en Gerencia Empre- \\ sarial, Doctor en Ciencias Gerenciales, Profesor de \\ Planta Universidad de La Guajira. \\ Correo electrónico: dlopezj@uniguajira.edu.co
}

Mirley Milena Salazar Morrón

Ingeniera ambiental de la Universidad de La Guajira. Asesora ambiental.

Información del artículo: recibido: noviembre de 2015, aceptado: enero de 2016

https://doi.org/10.17081/invinno.4.2.2486 


\section{Resumen}

E 1 presente trabajo de investigación se fundamenta en la evaluación de los impactos generados por la extracción de arcilla en la mina Santa Cruz (Manaure, La Guajira). El trabajo se realizó mediante la descripción y análisis de los impactos ambientales, socioculturales y económicos registrados a partir de visitas técnicas, entrevistas, encuestas y observaciones; posteriormente, todas estas técnicas fueron evaluadas a través de métodos cuantitativos como lista de chequeo, diagrama de redes, matriz de Leopold y matriz de valoración; relacionándolas con cada una de las actividades desarrolladas en las etapas del proyecto minero. La finalidad del trabajo consistió en generar un plan de manejo ambiental; en el que se contemplen medidas como: prevenir, mitigar, corregir o compensar las afectaciones a los recursos naturales intervenidos por la minería.

\begin{abstract}
This research work is based on assessment of impacts generated by the extraction of clay in Santa Cruz's mine, through description and analysis of environmental, sociocultural and economic impacts registered during technical visits, interviews, surveys and observations; subsequently evaluated by quantitative methods such as a checklist, network diagram, Leopold matrix rubric; making a direct relationship with each of the activities developed in the different stages of the mining project with the impact caused to the environmental components, the aim being to obtain an environmental management plan; in which are contemplated measures to prevent, mitigate, correct or compensate the damages made to natural resources operated by mining.
\end{abstract}

Palabras clave:

Evaluación de impacto

Ambiental

Extracción

Matriz de Leopold

Matriz de valoración,

Plan de manejo ambiental.

Keywords:

Environmental Impact

Assessment, extraction, Leopold matrix, rubric,

environmental management plan. 


\section{Introducción}

$\mathrm{L}$ a actividad minera es una de las que genera mayores ingresos económicos en Colombia, gracias a la diversidad de minerales que están presentes en el territorio nacional. El departamento de La Guajira es uno de los más privilegiados al respecto, lo que ha sido respaldado por estudios que comprueban la existencia de sal, yeso, caliza, carbón, arcilla, entre otros.

No obstante, en la actualidad se vienen desarrollando en la región una serie de conflictos socio-ambientales por causa de la explotación de los recursos naturales. Esto se debe en concreto a las prácticas de extracción ilegal de los minerales o al incumplimiento de las obligaciones ambientales y sociales derivadas de la legislación y de los instrumentos ambientales.

En efecto, no es posible garantizar el equilibrio entre desarrollo económico y gasto de oferta ambiental, si no existe un adecuado control estatal. La minería ilegal y/o artesanal, principalmente la dedicada a la extracción de arcilla en Colombia, ha venido creciendo en la última década, acarreando múltiples problemas ambientales, como la devastación de importantes ecosistemas, al no efectuarse ningún tipo de control $\mathrm{y} / \mathrm{o}$ actividad encaminada a prevenir, mitigar, corregir o compensar algún efecto negativo que esta actividad le genere al medio ambiente [1].

El caso de la extracción de arcilla en la comunidad Santa Cruz, municipio de Manaure, La Guajira, es importante porque los habitantes de este territorio y zonas aledañas ven esta actividad como su fuente principal de abastecimiento y empleo. Además, los trabajadores de esta actividad ancestral desconocen la le- gislación ambiental minera que rige este sector, así como los impactos ambientales que ocasionan al ejecutar su labor de minería artesanal; razón por la cual se debe intervenir, mediante la realización de estudios de evaluación de impacto ambiental para tomar las medidas de manejo frente a esta problemática.

Precisamente, el presente informe contiene la evaluación de impacto ambiental para la extracción de arcilla en Santa Cruz. De la misma forma, se propone un plan de manejo ambiental dirigidos a prevenir, mitigar, corregir o compensar los impactos ambientales generados por dicha actividad.

\section{Fundamento Teórico}

\section{Evaluación de impacto ambiental}

La evaluación de impacto ambiental implica el examen, análisis y evaluación de actividades programadas con el fin de garantizar desarrollo ecológicamente racional y sostenible; es una herramienta de planificación y gestión que permite identificar y predecir los efectos que se causarán sobre el ambiente, en razón de una actividad, obra o proyecto, cuantificándolos y ponderándolos para conducir a la toma de decisiones, dirigidas a prevenir, mitigar, corregir o compensar los impactos que un proyecto pueda producir sobre el medio [2].

Para realizar la evaluación de impacto ambiental se deben tener en cuenta ciertos criterios: que se identifiquen los impactos, que se establezca su relación, que se determine la relación de causa-efecto y se conozca la interacciones medio-acción que tendrá en cuenta la naturaleza, intensidad, extensión, momento, persistencia, reversibilidad, recuperabilidad, sinergia, acumulación, efecto y perio- 
dicidad; todo este proceso se determina mediante herramientas como la lista de chequeo (Check list), el diagrama de redes, la matriz de Leopold y la matriz de valoración.

\section{Plan de manejo ambiental}

Es el conjunto detallado de medidas y actividades que, producto de una evaluación ambiental, están orientadas a prevenir, mitigar, corregir o compensar los impactos y efectos ambientales debidamente identificados, que se causen por el desarrollo de un proyecto, obra o actividad. Incluye los planes de seguimiento, monitoreo, contingencia, y abandono según la naturaleza del proyecto, obra o actividad. [3].

Fichas de manejo ambiental La ficha de manejo es un documento que indica las particularidades y propiedades de una determinada actividad para su adecuado uso. En ella se identifica el área de influencia, determinando la línea base para predecir y valorar los posibles impactos ambientales; y así llegar a un plan de manejo ambiental que busque prevenir, mitigar, corregir o compensar los posibles impactos o efectos ambientales negativos que el proyecto pueda generar [4].

\section{Método}

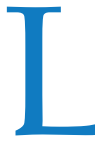
a investigación es de carácter descriptiva, por lo que se considera evaluar, especificar y analizar los diversos aspectos de la problemática ambiental y lo concerniente a la normatividad de la minería artesana [5].

Para el cumplimiento de los objetivos, el proyecto se desarrolló en tres (3) etapas: etapa de planeación, etapa preliminar y plan de evaluación.

a) Etapa de planeación: En su desarrollo, se identificó el área de estudio y se realizó una revisión documental, que posibilitó determinar las características históricas, espaciales, geográficas y culturales del área intervenida.

También se llevó a cabo una revisión de la normativa minera y ambiental, correspondiente al manejo ambiental minero, tomando en cuenta que la ilegalidad de la zona intervenida presupone el incumplimiento de la misma.

b) Etapa preliminar: En cumplimiento de esta, se practicó una visita técnica a la mina y se realizó la concertación con la autoridad tradicional para ejecutar las investigaciones pertinentes, en cuanto a observaciones y funcionamiento de la actividad minera.

Además, se seleccionaron herramientas evaluativas, a partir de los métodos clásicos para la realización de la evaluación de impacto ambiental.

c) Plan de evaluación: Incluyó: lista de chequeo, diagrama de redes, matriz de Leopold, matriz de valoración, fichas de manejo, programas ambientales, programa de monitoreo y seguimiento, plan de contingencia, plan de abandono y restauración final.

\section{Resultados y Análisis}

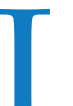

os impactos generados por la actividad minera artesanal, realizada en Santa Cruz, con el fin de extraer el mineral arcilloso característico de la región para la fabricación del ladrillo como producto final, afecta en gran medida los componentes bióticos, abióticos y socioeconómicos del lugar. Las actividades mineras agotan los recursos naturales y afectan al ecosistema predomínate en esta zona. Mediante la implementación de metodologías básicas de evaluación de impacto ambiental ya descritas (la lista de chequeo, diagrama de redes, ma- 
triz de Leopold, matriz de valoración y el plan de manejo), se presenta a continuación el efecto o alteración en el entorno. A través de la lista de chequeo se ordenaron los factores ambientales que se ven afectados por las actividades humanas. En esta lista los componentes se ubicaron jerárquicamente de mayor a menor según su grado de afectación, esta jerarquía se aplica al momento de establecer los impactos que se desencadenan en cada componente.

La idea es que los listados sean exhaustivos en la identificación de los impactos ambientales, pues su principal utilidad es rereportar todas las posibles consecuencias ligadas a la acción propuesta, asegurando, en una primera etapa de la evaluación, que ningún impacto relevante sea omitido. No puede haber un ejercicio de evaluación serio si no se empieza por hacer una lista de chequeo [6].

Con posterioridad a este procedimiento, se realizó el diagrama de redes que se fundamenta en analizar e integrar las causas de los efectos sobre el ambiente y los factores impactados por ellos. Los diagramas pueden ser realizados en forma genérica para cada tipo de actividad. En este caso, se orientó hacia las relaciones causa-efecto sobre las cuales deben poner atención o considerar en forma prioritaria adaptándolo a las condiciones particulares (naturales, sociales, económicas). 


\section{Lista de chequeo}

TABLA 1. Identificación de los impactos ambientales

\begin{tabular}{|c|c|c|c|c|}
\hline \multicolumn{2}{|c|}{ FACTOR AMBIENTAL } & \multirow{2}{*}{ IMPACTOS GENERADOS } & \multicolumn{2}{|c|}{ VALORACIÓN } \\
\hline MEDIO & COMPONENTE & & $(-)$ & $(+)$ \\
\hline \multirow{9}{*}{ ABIÓtICO } & \multirow{6}{*}{ SUELO } & Cambios del uso de suelo. & D & \\
\hline & & Cambios en la morfología del suelo. & D & \\
\hline & & Disgregación y desestabilización del suelo. & D & \\
\hline & & Alteración de las propiedades fisicoquímicas del suelo. & $\mathbf{O}$ & \\
\hline & & Erosión. & D & \\
\hline & & Aumento de la desertificación. & D & \\
\hline & \multirow{2}{*}{ AIRE } & Alteración de la calidad del aire por material particulado. & D & \\
\hline & & Emisión de gases invernadero $\left(\mathrm{CO}, \mathrm{CO}_{2}\right)$. & D & \\
\hline & AGUA & Desabastecimiento acuífero para las actividades prioritarias de la comunidad. & D & \\
\hline \multirow{5}{*}{ ВІÓTICO } & \multirow{5}{*}{ FAUNA Y FLORA } & $\begin{array}{l}\text { Pérdida de la vegetación correspondiente al matorral desértico, y el monte } \\
\text { espinoso subtropical. }\end{array}$ & D & \\
\hline & & Fragmentación de los ecosistemas (efecto barrera y efecto borde). & D & \\
\hline & & Desplazamiento de la fauna. & D & \\
\hline & & Aislamiento de las poblaciones. & D & \\
\hline & & Alteración del hábitat. & D & \\
\hline \multirow{6}{*}{$\begin{array}{l}\text { SOCIAL Y } \\
\text { CULTURAL }\end{array}$} & \multirow{2}{*}{ HUMANO } & Estético/Paisajístico. & & D \\
\hline & & Producción de enfermedades, por contaminación. & D & \\
\hline & \multirow{2}{*}{ SOCIAL Y CULTURAL } & Cambios de las actividades productivas de la comunidad wayuu. & & $\mathbf{0}$ \\
\hline & & Aumento en la diversidad étnica y cultural. & & O \\
\hline & \multirow{2}{*}{ ECÓNOMICO } & Generación de empleo. & & D \\
\hline & & Arrendamiento/Venta de terrenos. & & O \\
\hline
\end{tabular}




\section{Diagrama de redes}

\section{TABLA 2. Diagrama de redes}




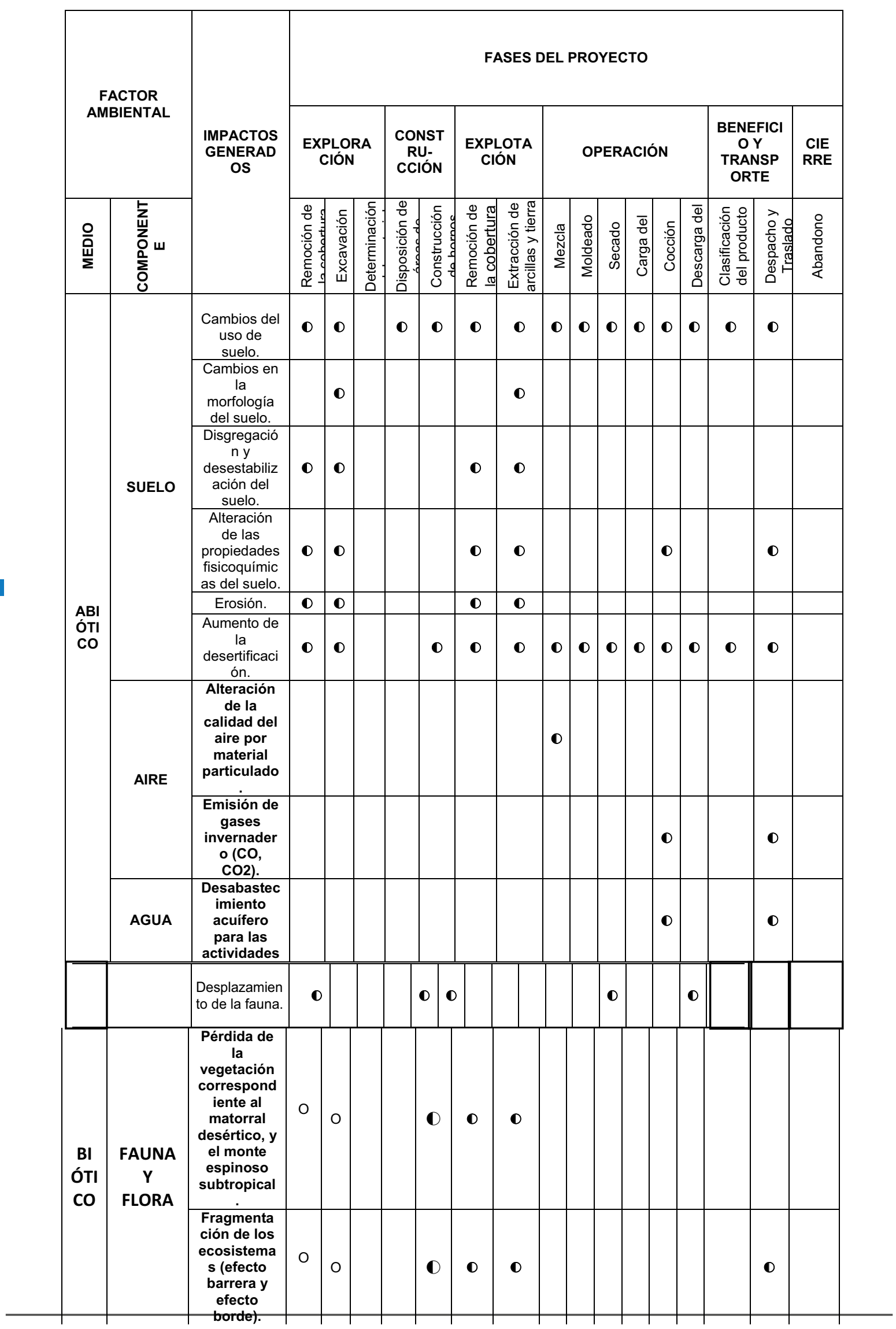

Articulo de Investigación 


\begin{tabular}{|c|c|c|c|c|c|c|c|c|c|c|c|c|c|c|c|c|c|c|}
\hline & & $\begin{array}{l}\text { Aislamiento } \\
\text { de las } \\
\text { poblaciones. }\end{array}$ & & & & & & & & & & & & & & & & \\
\hline & & $\begin{array}{l}\text { Alteración del } \\
\text { hábitat. }\end{array}$ & D & D & & & D & D & D & & & & & D & & & D & \\
\hline \multirow{6}{*}{$\begin{array}{l}\text { SOCIA } \\
\text { LY } \\
\text { CULTU } \\
\text { RAL }\end{array}$} & \multirow[b]{2}{*}{$\begin{array}{c}\text { HUMAN } \\
0\end{array}$} & $\begin{array}{c}\text { Estético/Paisa } \\
\text { jístico. }\end{array}$ & & & & & 0 & D & & & & & & & & & & D \\
\hline & & $\begin{array}{c}\text { Producción de } \\
\text { enfermedades } \\
\text {, por } \\
\text { contaminació } \\
\text { n. }\end{array}$ & & & & & & & & & & & & 0 & & & & \\
\hline & \multirow[t]{2}{*}{$\begin{array}{c}\text { SOCIAL } \\
Y \\
\text { CULTUR } \\
\text { AL }\end{array}$} & $\begin{array}{l}\text { Cambios de } \\
\text { las } \\
\text { actividades } \\
\text { productivas } \\
\text { de la } \\
\text { comunidad } \\
\text { wayuu. }\end{array}$ & $\mathbf{D}$ & D & $\mathbf{D}$ & D & D & $\mathbf{0}$ & $\mathbf{D}$ & D & D & D & D & $\mathbf{0}$ & $\mathbf{D}$ & $\mathbf{0}$ & $\mathbf{D}$ & \\
\hline & & $\begin{array}{l}\text { Aumento en } \\
\text { la diversidad } \\
\text { étnica y } \\
\text { cultural. }\end{array}$ & D & D & D & D & 0 & D & D & D & D & D & D & $\mathbf{0}$ & $\mathbf{D}$ & O & $\mathbf{D}$ & \\
\hline & \multirow{2}{*}{$\begin{array}{l}\text { ECÓNO } \\
\text { MICO }\end{array}$} & $\begin{array}{l}\text { Generación } \\
\text { de empleo. }\end{array}$ & D & D & D & D & 0 & D & D & D & D & D & D & $\mathbf{0}$ & $\mathbf{D}$ & D & 0 & \\
\hline & & $\begin{array}{l}\text { Arrendamient } \\
\text { o/Venta de } \\
\text { terrenos. }\end{array}$ & & & & D & & & & & & & & & & & & \\
\hline
\end{tabular}

\section{Matriz de Leopold}

TABLA 3. Resultados de la evaluación de los impactos ambientales 


\begin{tabular}{|c|c|c|c|c|c|c|c|c|c|c|c|c|c|c|c|c|c|c|c|}
\hline \multirow{2}{*}{\multicolumn{2}{|c|}{$\begin{array}{c}\text { FACTOR } \\
\text { AMBIENTAL }\end{array}$}} & \multirow{3}{*}{$\begin{array}{l}\text { IMPACTOS } \\
\text { GENERADO } \\
\mathbf{S}\end{array}$} & \multicolumn{17}{|c|}{ FASES DEL PROYECTO } \\
\hline & & & \multicolumn{2}{|c|}{$\underset{\mathbf{N}}{\text { EXPLORACIÓ }}$} & \multicolumn{3}{|c|}{ CONSTRUCCIÓN } & \multicolumn{2}{|c|}{$\begin{array}{l}\text { EXPLOTA } \\
\text { CIÓN }\end{array}$} & \multicolumn{6}{|c|}{ OPERACIÓN } & \multicolumn{2}{|c|}{$\begin{array}{l}\text { BENEFICI } \\
\text { OY } \\
\text { TRANSPO } \\
\text { RTE } \\
\end{array}$} & \multirow{2}{*}{$\begin{array}{c}\begin{array}{c}\text { CIERR } \\
\text { E }\end{array} \\
\\
\\
\stackrel{0}{0} \\
\frac{0}{0} \\
\frac{\pi}{\pi} \\
\stackrel{0}{<}\end{array}$} & \multirow[t]{2}{*}{ TOTAL } \\
\hline MEDIO & $\begin{array}{l}\text { COMPO } \\
\text { NENTE }\end{array}$ & & 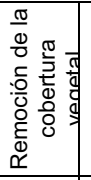 & 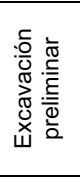 & 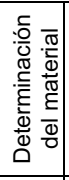 & 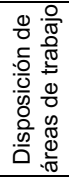 & 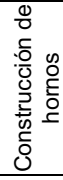 & 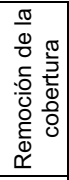 & 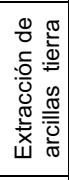 & $\begin{array}{l}\frac{\pi}{0} \\
N \\
\sum\end{array}$ & $\begin{array}{l}\frac{0}{0} \\
\frac{\pi}{0} \\
\frac{0}{0} \\
\sum\end{array}$ & $\begin{array}{l}\frac{0}{8} \\
\text { ס্ } \\
\infty \\
\infty\end{array}$ & 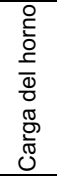 & 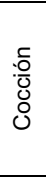 & 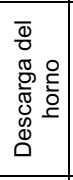 & 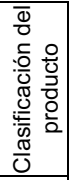 & 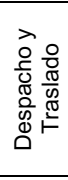 & & \\
\hline \multirow{12}{*}{$\begin{array}{c}\text { ABIÓTIC } \\
0\end{array}$} & \multirow{12}{*}{ SUELO } & \multirow{2}{*}{$\begin{array}{l}\text { Cambios del } \\
\text { uso de suelo. }\end{array}$} & -10 & -10 & -10 & -5 & -1 & -1 & -1 & $\begin{array}{c}- \\
10 \\
\end{array}$ & $\begin{array}{c}- \\
10 \\
\end{array}$ & 10 & -10 & 10 & -10 & -10 & -10 & -1 & -119 \\
\hline & & & 1 & 1 & 1 & 10 & 10 & 10 & 10 & 10 & 10 & 10 & 10 & 10 & 10 & 5 & 5 & 10 & 123 \\
\hline & & \multirow{2}{*}{$\begin{array}{l}\text { Cambios en } \\
\text { la morfología } \\
\text { del suelo. }\end{array}$} & -10 & -10 & -10 & -10 & -1 & -1 & -1 & $\overline{10}$ & $\begin{array}{c} \\
10\end{array}$ & $\begin{array}{c}- \\
10\end{array}$ & -10 & -5 & -10 & -10 & -10 & -1 & -119 \\
\hline & & & 1 & 1 & 1 & 10 & 10 & 10 & 10 & 10 & 10 & 10 & 10 & 10 & 10 & 5 & 5 & 10 & 123 \\
\hline & & \multirow{2}{*}{$\begin{array}{c}\text { Disgregación } \\
y \\
\text { desestabilizac } \\
\text { ión del suelo. }\end{array}$} & -10 & -10 & -10 & -10 & -5 & -1 & -1 & $\begin{array}{c}- \\
10\end{array}$ & $\begin{array}{c}- \\
10\end{array}$ & $\begin{array}{c}- \\
10 \\
\end{array}$ & -10 & -5 & -10 & -10 & -10 & -1 & -123 \\
\hline & & & 1 & 1 & 1 & 10 & 10 & 10 & 10 & 10 & 10 & 10 & 10 & 10 & 10 & 5 & 5 & 10 & 123 \\
\hline & & \multirow{2}{*}{$\begin{array}{c}\text { Alteración de } \\
\text { las } \\
\text { propiedades } \\
\text { fisicoquímicas } \\
\text { del suelo. } \\
\end{array}$} & -10 & -10 & -10 & -5 & -5 & -1 & -1 & $\begin{array}{c}- \\
10\end{array}$ & $\begin{array}{c}- \\
10\end{array}$ & $\overline{10}$ & -10 & -1 & -10 & -10 & -10 & -1 & -114 \\
\hline & & & 1 & 1 & 1 & 10 & 10 & 10 & 10 & 10 & 10 & 10 & 10 & 10 & 10 & 5 & 5 & 10 & 123 \\
\hline & & \multirow{2}{*}{ Erosión. } & -10 & -10 & -10 & -5 & -5 & -1 & -1 & $\begin{array}{c}- \\
10 \\
\end{array}$ & $\begin{array}{c}- \\
10\end{array}$ & $\begin{array}{c}- \\
10 \\
\end{array}$ & -10 & $\begin{array}{c}- \\
10\end{array}$ & -10 & -10 & -10 & -1 & -123 \\
\hline & & & 1 & 1 & 1 & 10 & 10 & 10 & 10 & 10 & 10 & 10 & 10 & 10 & 10 & 5 & 5 & 10 & 123 \\
\hline & & \multirow{2}{*}{$\begin{array}{c}\text { Aumento de } \\
\text { la } \\
\text { desertificació } \\
\mathrm{n} .\end{array}$} & -10 & -10 & -10 & -5 & -5 & -1 & -1 & $\begin{array}{c}- \\
10\end{array}$ & $\begin{array}{l}- \\
10\end{array}$ & - & -10 & $\overline{10}$ & -10 & -10 & -10 & -1 & -123 \\
\hline & & & 1 & 1 & 1 & 10 & 10 & 10 & 10 & 10 & 10 & 10 & 10 & 10 & 10 & 5 & 5 & 10 & 123 \\
\hline
\end{tabular}

\begin{tabular}{|c|c|c|c|c|c|c|c|c|c|c|c|c|c|c|c|c|c|c|c|}
\hline & \multirow{4}{*}{ AIRE } & \multirow{2}{*}{$\begin{array}{l}\text { Alteración de la } \\
\text { calidad del aire } \\
\text { por material } \\
\text { particulado. }\end{array}$} & -10 & -10 & -10 & -10 & -10 & -10 & -10 & -5 & -10 & -5 & -5 & -1 & -10 & -10 & -5 & -10 & -131 \\
\hline & & & 1 & 1 & 1 & 10 & 10 & 10 & 10 & 10 & 10 & 10 & 10 & 10 & 10 & 5 & 5 & 10 & 123 \\
\hline & & \multirow{2}{*}{$\begin{array}{l}\text { Emisión de gases } \\
\text { invernadero }(\mathrm{CO}, \\
\left.\qquad \mathrm{CO}_{2}\right) .\end{array}$} & -10 & -10 & -10 & -10 & -10 & -10 & -10 & -10 & -10 & -10 & -10 & -1 & -10 & -10 & -5 & -1 & -137 \\
\hline & & & 1 & 1 & 1 & 10 & 10 & 10 & 10 & 10 & 10 & 10 & 10 & 10 & 10 & 5 & 5 & 10 & 123 \\
\hline & \multirow{2}{*}{ AGUA } & \multirow{2}{*}{$\begin{array}{c}\text { Desabastecimient } \\
\text { o acuífero. }\end{array}$} & -10 & -10 & -10 & -10 & -5 & -10 & -10 & -10 & -10 & -10 & -10 & - & -10 & -10 & -10 & -1 & -146 \\
\hline & & & 1 & 1 & 1 & 10 & 10 & 10 & 10 & 10 & 10 & 10 & 10 & 10 & 10 & 5 & 5 & 10 & 123 \\
\hline \multirow{10}{*}{$\begin{array}{l}\text { BIÓTIC } \\
\text { O }\end{array}$} & \multirow{10}{*}{$\begin{array}{c}\text { FAUNA Y } \\
\text { FLORA }\end{array}$} & \multirow{2}{*}{$\begin{array}{c}\text { Pérdida de la } \\
\text { vegetación } \\
\text { correspondiente al } \\
\text { matorral } \\
\text { desértico, y el } \\
\text { monte espinoso } \\
\text { subtropical. } \\
\end{array}$} & -10 & -10 & -10 & -5 & -10 & -1 & -10 & -10 & -10 & -10 & -10 & 10 & -10 & -10 & -10 & -1 & -137 \\
\hline & & & 1 & 1 & 1 & 10 & 10 & 10 & 10 & 10 & 10 & 10 & 10 & 10 & 10 & 5 & 5 & 10 & 123 \\
\hline & & \multirow{2}{*}{$\begin{array}{l}\text { Fragmentación de } \\
\text { los ecosistemas } \\
\text { (efecto barrera y } \\
\text { efecto borde). }\end{array}$} & -10 & -10 & -10 & -1 & -5 & -1 & -5 & -10 & -10 & -10 & -10 & 10 & -10 & -10 & -10 & -1 & -123 \\
\hline & & & 1 & 1 & 1 & 10 & 10 & 10 & 10 & 10 & 10 & 10 & 10 & 10 & 10 & 5 & 5 & 10 & 123 \\
\hline & & \multirow{2}{*}{$\begin{array}{l}\text { Desplazamiento } \\
\text { de la fauna. }\end{array}$} & -10 & -10 & -10 & -1 & -5 & -1 & -5 & -10 & -10 & -10 & -10 & $\overline{10}$ & -10 & -10 & -10 & -1 & -123 \\
\hline & & & 1 & 1 & 1 & 10 & 10 & 10 & 10 & 10 & 10 & 10 & 10 & 10 & 10 & 5 & 5 & 10 & 123 \\
\hline & & \multirow{2}{*}{$\begin{array}{l}\text { Aislamiento de las } \\
\text { poblaciones. }\end{array}$} & -10 & -10 & -10 & -5 & -5 & -5 & -5 & -10 & -10 & -10 & -10 & 10 & -10 & -10 & -10 & -1 & -131 \\
\hline & & & 1 & 1 & 1 & 10 & 10 & 10 & 10 & 10 & 10 & 10 & 10 & 10 & 10 & 5 & 5 & 10 & 123 \\
\hline & & \multirow{2}{*}{$\begin{array}{l}\text { Alteración del } \\
\text { hábitat. }\end{array}$} & -10 & -10 & -10 & -5 & -5 & -1 & -1 & -10 & -10 & -10 & -10 & $\overline{10}$ & -10 & -10 & -10 & -1 & -123 \\
\hline & & & 1 & 1 & 1 & 10 & 10 & 10 & 10 & 10 & 10 & 10 & 10 & 10 & 10 & 5 & 5 & 10 & 123 \\
\hline
\end{tabular}




\begin{tabular}{|c|c|c|c|c|c|c|c|c|c|c|c|c|c|c|c|c|c|c|c|}
\hline \multirow{12}{*}{$\begin{array}{c}\text { SOCIAL Y } \\
\text { CULTURA } \\
\text { L }\end{array}$} & \multirow{4}{*}{ HUMANO } & \multirow{2}{*}{$\begin{array}{l}\text { Estético/Paisaj } \\
\text { istico. }\end{array}$} & -10 & -10 & -10 & -1 & -1 & -1 & -1 & -10 & -10 & -10 & -10 & -5 & -5 & -10 & -10 & -1 & -105 \\
\hline & & & 1 & 1 & 1 & 10 & 10 & 10 & 10 & 10 & 10 & 10 & 10 & 10 & 10 & 5 & 5 & 10 & 123 \\
\hline & & \multirow{2}{*}{$\begin{array}{l}\text { Producción de } \\
\text { enfermedades, } \\
\text { por } \\
\text { contaminación }\end{array}$} & -10 & -10 & -10 & -10 & -10 & -10 & -10 & -10 & -10 & -10 & -10 & -1 & -5 & -10 & -10 & -1 & -137 \\
\hline & & & 1 & 1 & 1 & 10 & 10 & 10 & 10 & 10 & 10 & 10 & 10 & 10 & 10 & 5 & 5 & 10 & 123 \\
\hline & \multirow{4}{*}{$\begin{array}{c}\text { SOCIAL Y } \\
\text { CULTURAL }\end{array}$} & \multirow{2}{*}{$\begin{array}{l}\text { Cambios de } \\
\text { las actividades } \\
\text { productivas de } \\
\text { la comunidad } \\
\text { wayuu. }\end{array}$} & 1 & 1 & 1 & 5 & 5 & 5 & 5 & 5 & 5 & 5 & 5 & 5 & 5 & 10 & 10 & 1 & 74 \\
\hline & & & 1 & 1 & 1 & 10 & 10 & 10 & 10 & 10 & 10 & 10 & 10 & 10 & 10 & 5 & 5 & 10 & 123 \\
\hline & & \multirow{2}{*}{$\begin{array}{l}\text { Aumento en la } \\
\text { diversidad } \\
\text { étnica y } \\
\text { cultural. }\end{array}$} & 1 & 1 & 1 & 5 & 5 & 5 & 5 & 5 & 5 & 5 & 5 & 5 & 5 & 5 & 5 & $\overline{10}$ & 53 \\
\hline & & & 1 & 1 & 1 & 10 & 10 & 10 & 10 & 10 & 10 & 10 & 10 & 10 & 10 & 5 & 5 & 5 & 118 \\
\hline & \multirow{4}{*}{$\begin{array}{c}\text { ECONÓMIC } \\
0\end{array}$} & \multirow{2}{*}{$\begin{array}{l}\text { Generación de } \\
\text { empleo. }\end{array}$} & 1 & 1 & 1 & 10 & 10 & 10 & 10 & 10 & 10 & 10 & 10 & 10 & 10 & 10 & 10 & 10 & 113 \\
\hline & & & 1 & 1 & 1 & 10 & 10 & 10 & 10 & 10 & 10 & 10 & 10 & 10 & 10 & 5 & 5 & 5 & 118 \\
\hline & & \multirow{2}{*}{$\begin{array}{l}\text { Arrendamiento } \\
\text { /Venta de } \\
\text { terrenos. }\end{array}$} & 1 & 1 & 1 & 10 & 10 & 10 & 10 & 10 & 10 & 10 & 10 & 10 & 10 & 10 & -10 & 1 & 104 \\
\hline & & & 1 & 1 & 1 & 10 & 10 & 10 & 10 & 10 & 10 & 10 & 10 & 10 & 10 & 5 & 5 & 1 & 114 \\
\hline \multicolumn{3}{|c|}{ TOTAL. } & $\overline{136}$ & $\overline{136}$ & - & 132 & 142 & 174 & 157 & 75 & 70 & 75 & 75 & $\begin{array}{c}12 \\
1\end{array}$ & 80 & -25 & -35 & $\begin{array}{c}13 \\
8\end{array}$ & \\
\hline
\end{tabular}

Tablas 4.

Rangos de los valores de la magnitud e importancia para la EIA de valoración.

\begin{tabular}{|c|c|c|}
\hline \multirow{2}{*}{ Rango } & \multicolumn{2}{|c|}{ Magnitud } \\
\cline { 2 - 3 } & Positivo (+) N egativo (-) \\
\hline Alto & 101 & \\
\hline Medio & 5 & 5 \\
\hline Bajo & 1 & 10 \\
\hline
\end{tabular}

\begin{tabular}{|c|c|}
\hline Rango & Importancia \\
\hline Alto & 10 \\
\hline Medio & 5 \\
\hline Bajo & 1 \\
\hline
\end{tabular}




\section{Matriz}

Tabla 5. Matriz de valoración (Impactos significativo en cada componente).

\begin{tabular}{|c|c|c|c|c|c|c|c|c|c|c|c|c|c|c|}
\hline \multirow{2}{*}{\multicolumn{2}{|c|}{ FACTOR AMBIENTAL }} & \multirow{3}{*}{$\begin{array}{l}\text { IMPACTOS } \\
\text { GENERADOS }\end{array}$} & \multicolumn{12}{|c|}{ VALORACIÓN } \\
\hline & & & \multirow{2}{*}{$\begin{array}{l}\frac{\mathbb{N}}{\mathbb{N}} \\
\frac{\pi}{\sqrt[N]{\pi}} \\
\frac{\pi}{z}\end{array}$} & \multirow{2}{*}{ 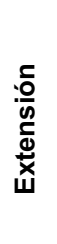 } & \multirow{2}{*}{ 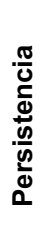 } & \multirow{2}{*}{$\frac{\frac{\pi}{0}}{\frac{\pi}{0}}$} & \multirow{2}{*}{ 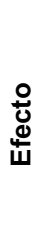 } & \multirow{2}{*}{ 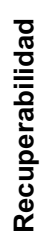 } & \multirow{2}{*}{ 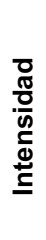 } & \multirow{2}{*}{ 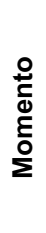 } & \multirow{2}{*}{ 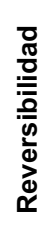 } & \multirow{2}{*}{ 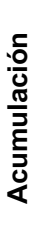 } & \multirow{2}{*}{$\begin{array}{l}\frac{0}{\pi} \\
\frac{\pi}{0} \\
\frac{0}{0} \\
\frac{0}{0} \\
\frac{0}{0} \\
0 \\
0\end{array}$} & \multirow{2}{*}{ 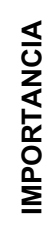 } \\
\hline MEDIO & $\begin{array}{l}\text { COMPO } \\
\text { NENTE }\end{array}$ & & & & & & & & & & & & & \\
\hline \multirow{7}{*}{ ABIÓTICO } & \multirow{6}{*}{ SUELO } & $\begin{array}{c}\text { Cambios del uso de } \\
\text { suelo. }\end{array}$ & $(-)$ & 1 & 4 & 2 & 4 & 4 & 4 & 4 & 2 & 1 & 2 & 37 \\
\hline & & $\begin{array}{c}\text { Cambios en la morfología } \\
\text { del suelo. }\end{array}$ & $(-)$ & 1 & 4 & 4 & 4 & 2 & 8 & 4 & 4 & 4 & 2 & 54 \\
\hline & & $\begin{array}{c}\text { Disgregación y } \\
\text { desestabilización del } \\
\text { suelo. }\end{array}$ & $(-)$ & 2 & 4 & 4 & 4 & 4 & 2 & 4 & 4 & 4 & 2 & 40 \\
\hline & & $\begin{array}{c}\text { Alteración de las } \\
\text { propiedades } \\
\text { fisicoquímicas del suelo. }\end{array}$ & $(-)$ & 1 & 4 & 4 & 4 & 2 & 4 & 4 & 4 & 4 & 2 & 42 \\
\hline & & Erosión. & $(-)$ & 2 & 2 & 2 & 4 & 2 & 8 & 4 & 4 & 4 & 2 & 52 \\
\hline & & $\begin{array}{l}\text { Aumento de la } \\
\text { desertificación. }\end{array}$ & $(-)$ & 1 & 2 & 2 & 4 & 2 & 4 & 1 & 4 & 4 & 2 & 35 \\
\hline & AIRE & $\begin{array}{l}\text { Alteración de la calidad } \\
\text { del aire por material } \\
\text { particulado. }\end{array}$ & $(-)$ & 2 & 2 & 2 & 1 & 1 & 1 & 4 & 1 & 4 & 1 & 23 \\
\hline \multirow{3}{*}{ ВіÓтICO } & \multirow{3}{*}{$\begin{array}{l}\text { FAUNA } \\
\text { Y } \\
\text { FLORA }\end{array}$} & $\begin{array}{l}\text { Fragmentación de los } \\
\text { ecosistemas (efecto } \\
\text { barrera y efecto borde). }\end{array}$ & $(-)$ & 1 & 2 & 2 & 4 & 2 & 2 & 4 & 2 & 4 & 2 & 30 \\
\hline & & $\begin{array}{l}\text { Desplazamiento de la } \\
\text { fauna. }\end{array}$ & $(-)$ & 2 & 1 & 2 & 4 & 2 & 4 & 4 & 2 & 1 & 2 & 34 \\
\hline & & Alteración del hábitat. & $(-)$ & 1 & 2 & 4 & 4 & 4 & 2 & 4 & 2 & 1 & 2 & 31 \\
\hline $\begin{array}{c}\text { SOCIAL Y } \\
\text { CULTURA } \\
\text { L }\end{array}$ & $\begin{array}{c}\text { HUMAN } \\
0\end{array}$ & Estético/Paisajístico. & $(-)$ & 2 & 4 & 4 & 4 & 4 & 8 & 2 & 4 & 4 & 2 & 56 \\
\hline
\end{tabular}




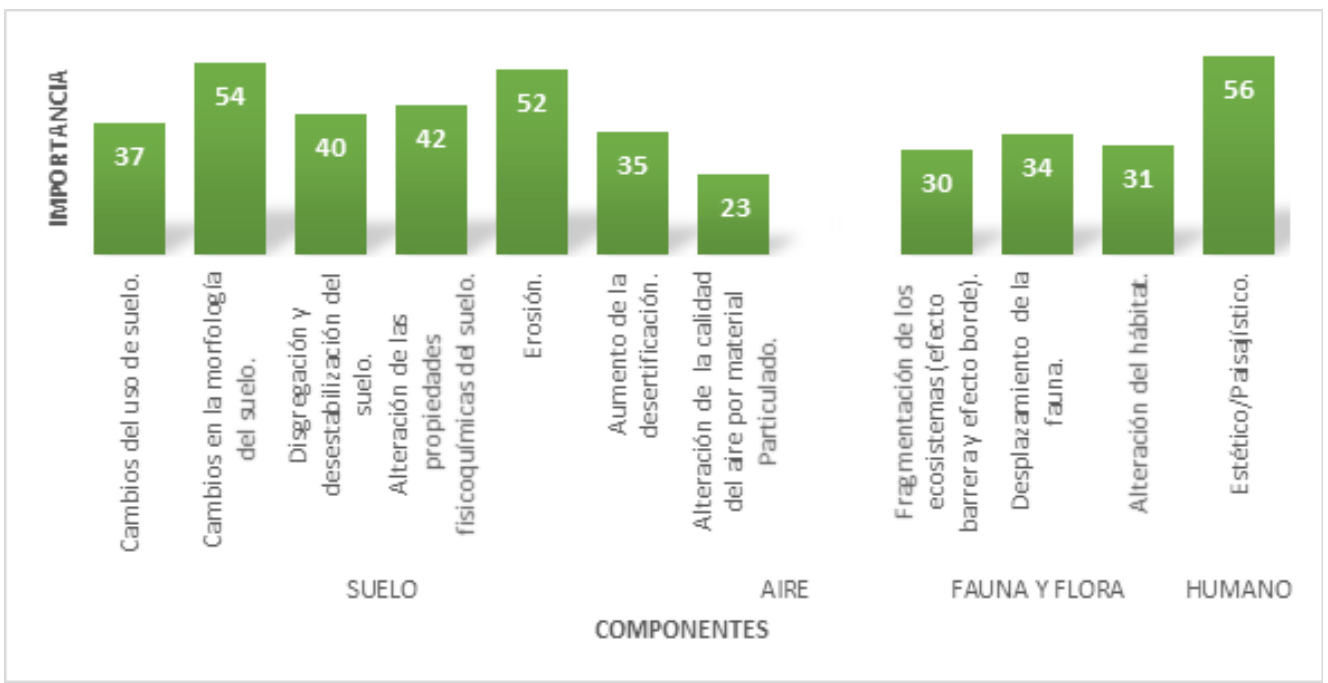

Grafico 1. Representación de los impactos ambientales

\section{Tabla 6. Rangos de calificación de la matriz de valoración}

De acuerdo con los análisis realizados en la matriz de Leopold, los componentes de impacto significativo pasaron a una segunda evaluación minuciosa para destacar los impactos de mayor relevancia.

Se obtuvo así que los impactos en los medios biótico, abiótico, social y cultural fueron de naturaleza negativa. A partir del resultado de la importancia, se calificaron finalmente: 3 impactos severos, 1 impacto irrelevante y 7 impactos moderados. Los impactos moderados alteran los componentes suelo, aire, fauna y flora, a excepción del componente humano en el cual la afectación es severa.

Suelo. Es el componente más afectado por ser la base de las actividades mineras. En consecuencia, los impactos más relevantes son los cambios en la morfología del suelo y la erosión; esto se debe al retiro de la cobertura vegetal y extracción del suelo. Por otra parte, e resulta-

\begin{tabular}{|c|c|}
\hline Irrelevante & $<\mathbf{2 5}$ \\
\hline Moderado & $\mathbf{2 5} \mathbf{y}<\mathbf{5 0}$ \\
\hline Severo & $>\mathbf{5 0}$ \\
\hline do de los impactos antes mencionados
\end{tabular}
ocasiona un impacto estético/paisajístico que puede depreciar el valor en la propiedad.

Aire. La alteración de la calidad del aire por material particulado es el único impacto irrelevante. De hecho, es el impacto menos notable, debido a que solo se ocasiona durante las actividades de cocción, secado, despacho y traslado. Aunque aparece en todas estas etapas, se debe tener en cuenta que durante la cocción, el horno se encuentra casi sellado en su totalidad, solo se presentan pequeños escapes durante el secado. Este material particulado se emite también en pequeña proporción al momento de acarrear los ladrillos hacia el área de secado, así como durante el despacho y traslado porque la carga de ladrillos no posee cobertura, y el movimiento del vehículo en el que se transporta ocasiona el impacto mencionado. 
Fauna y flora. El componente biótico de la zona es impactado directa e indirectamente en las etapas del proceso minero, exponiendo las interacciones ecológicas a nuevas adaptaciones.

Agua. En el componente agua, no se logró identificar un impacto ambiental significativo; la comunidad obtiene el agua a través de una estructura subterránea para su consumo y este suministro de agua no genera costos a los propietarios de las minas ni a la comunidad, debido a que el pozo fue donado por el municipio de Manaure a las comunidades Wayuu. Sin embargo, la extracción del recurso agua puede tornarse en un problema ambiental si se abusa de ello, por lo tanto, si no se toman precauciones en el manejo del agua subterránea, en algún momento esta podría escasear, provocando una calamidad ambiental y social, ya que el acuífero es la única fuente que abastece de este recurso vital a la comunidad.

Humano. En este componente se obtuvo un impacto severo y la mayor afectación ambiental en lo estético/paisajístico, que deteriora el aspecto visual en los escenarios naturales del resguardo indígena, impidiendo a los habitantes de la comunidad el goce de un ambiente sano.

El Plan de Manejo Ambiental (PMA) es la última actividad desarrollada para finiquitar y dar cumplimiento a la evaluación de impacto ambiental. Este se orienta a prevenir, mitigar, corregir o compensar los impactos y efectos ambientales debidamente identificados, que se causen por el desarrollo de un proyecto, obra o actividad.

Para garantizar el desempeño de las acciones y propuestas en el plan de manejo ambiental realizado en la mina artesanal de la comunidad Santa Cruz, los parámetros que se tuvieron en cuenta en el programa de monitoreo y seguimiento fueron: seguimiento y monitoreo interno, vigilancia comunitaria e información a la comunidad.

Como última medida se realizó el plan de abandono y restauración final. En este aspecto se determinaron las acciones encaminadas a las medidas de manejo ambiental, llevando como línea base las directrices abordadas en la metodología general para la presentación de estudios ambientales en Colombia. Todo ello con el objetivo de garantizar un ambiente adecuado y sostenible en la comunidad tradicional indígena wayuu Santa Cruz.

Las medidas del plan de abandono y restauración final contemplan principal21 mente al desmantelamiento y abandono, la limpieza del sitio y la restauración de las zonas.

\section{Conclusiones}

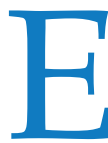
1 componente con un mayor grado de impacto es el suelo, en el cual se manifiestan cambios en la morfología del mismo, quedando clasificados como impactos severos dentro de los rangos de evaluación de la matriz de importancia.

La actividad minera para la realización de ladrillos es un eslabón muy importante en el ámbito socioeconómico, teniendo en cuenta que esta genera empleo y sustento a las familias asentadas en la comunidad de Santa Cruz. No obstante, la ladrillera conformada se encuentra en la ilegalidad: no cuenta con los requisitos jurídicos establecidos como el del título minero y la 
licencia ambiental, que son de vital importancia para la ejecución de dicha actividad, teniendo en cuenta la normatividad vigente colombiana.

Existe un documento suscrito entre el Ministerio de Minas y Energía y la Corporación Autónoma Regional de La Guajira, pero el mismo exige mayores requisitos según la formalización minera detallada en el decreto 0933 de 2013 del Ministerio de Minas y Energía.

Siendo la actividad minera de tipo artesanal, su tecnificación es poca, lo que dificulta el aumento de la producción y disminuye los niveles de desarrollo. Las medidas de capacitación minero-ambiental y de seguridad del personal son muy importantes, porque en muchos casos la contaminación ambiental es causada por prácticas inadecuadas en el desarrollo de las actividades. De igual forma, estas capacitaciones pueden evitar la generación de accidentes.

En la comunidad de desarrollan 4 tipos de actividades económicas, y la producción de artesanías es la que mayormente se realiza, seguida por la minería. Esta última es, por otro lado, la actividad que más ingresos y empleo genera en la comunidad.

La demanda de ladrillos en el año 2016 aumentó con respecto al año 2015, lo que supone un aumento en la industria de la construcción.

Las minas ubicadas en la zona de inundación del río se afectan por los fenómenos climáticos, teniendo en cuenta que en épocas de invierno las producciones se detienen por inundación del área de trabajo. De igual forma, la producción se afecta cuando hay sequía debido a la necesidad de agua que se tiene para las labores de mezclado.

A largo plazo se prevé que la minería no es viable, ya que el cambio climático y la escasez de agua se agudizan con el tiempo y, a futuro, el agua disponible se utilizará primordialmente para el consumo humano.

La minería ha aportado mejores condiciones económicas a la comunidad, aumento en la población local y flotante, nuevas viviendas, ampliación de la diversidad cultural y se incluyó como nueva actividad dentro de las desarrolladas tradicionalmente.

La actividad minera se encuentra en auge, lo que se evidencia en la construcción de más hornos y de excavaciones en nuevas áreas. Además, la minería fue una pieza clave para el abandono de las actividades delictivas que se desarrollaban en el sector antes de que esta actividad existiera.

Por todo lo anterior, la minería no puede verse como un elemento negativo, pero tampoco puede aplicarse bajo los principios del desarrollo sostenible. Más bien, esta actividad económica puede desarrollarse de manera controlada y responsable con el medio ambiente, protegiendo y rehabilitando las zonas intervenidas.

\section{Referencias Bibliográficas}

[1] Serafín, "Evaluación cualitativa del impacto ambiental generado por la actividad minera en la rinconada puno" piura, peru. Disponible en: http://pirhua.udep. edu.pe/bitstream/handle/123456789/1482/ MAS_GAA_007.pdf?sequence=1, 2009. 
[2] Holcim, "Guía de gestión ambiental para minería no metálica”. Disponible en: https://cmsdata.iucn.org/downloads/guia_ mineria.pdf, 2009.

[3] L. García, "Aplicación del análisis multicriterio en la evaluación de impactos ambientales", Disponible en: http://www.tdx.cat/bitstream/handle/10803/6830/01Lagl01, 2004.

[4] MADS - Decreto número 2041, 2015. 2

[5] Martínez, J. "Métodos de investigación cualitativa", Revista de la Corporación Internacional para el Desarrollo Educativo. Disponible en: http://www.cide.edu. $\mathrm{co} / \mathrm{doc} /$ investigacion/3.\%20 metodos $\% 20$ de\%20investigacion.pdf, 2011.

[6] Rojas, "Univrsidad de San Carlos de Guatemala, manual de evaluacion de impacto ambiental" Disponible en: http:// biblioteca.usac.edu.gt/tesis/08/08_1163_ IN.pdf. pág. 76, 2003.

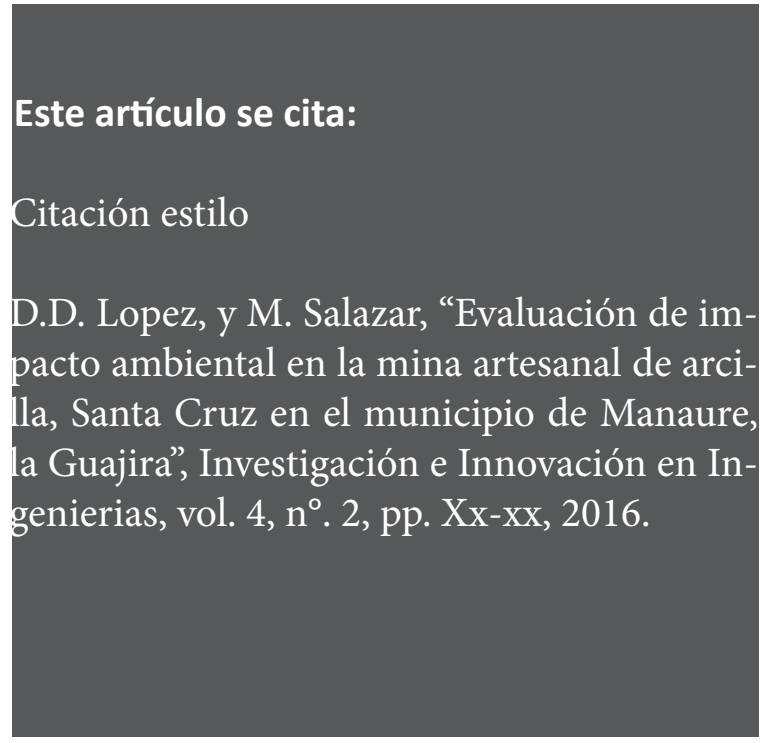

